\title{
Surface-Micromachined Parylene Dual Valves for On-Chip Unpowered Microflow Regulation
}

\author{
Po-Jui Chen, Student Member, IEEE, Student Member, ASME, Damien C. Rodger, Student Member, IEEE, \\ Ellis M. Meng, Member, IEEE, Mark S. Humayun, Member, IEEE, and Yu-Chong Tai, Fellow, IEEE
}

\begin{abstract}
This paper presents the world's first surface-micromachined parylene dual-valved microfluidic system for on-chip unpowered microflow regulation. Incorporating a normally closed and a normally open passive check valve in a back-to-back configuration inside a microchannel, the dual-valved system has successfully regulated the pressure/flow rate of air and liquid without power consumption or electronic/magnetic/thermal transduction. By exclusively using parylene $C$ (poly-para-xylylene $C$ ) as the structural material, the fabricated valves have higher flexibility to shunt flows in comparison to other conventional thin-film valves. A state-of-the-art multilayer polymer surface-micromachining technology is applied here to fabricate parylene microvalves of various designs. The parylene-based devices are completely biocompatible/implantable and provide an economical paradigm for fluidic control in integrated lab-on-a-chip systems. Design, fabrication, and characterization of the parylene dual valves are discussed in this paper. Testing results have successfully demonstrated that the microflow regulation of the on-chip dual-valved system can achieve a bandpass profile in which the pressure control range is 0-50 mmHg with corresponding flow rates up to $2 \mathrm{~mL} / \mathrm{min}$ for air flow and $1 \mu \mathrm{L} / \mathrm{min}$ flow rate for water flow. This regulation range is suitable for controlling biological conditions in human health care, with potential applications including drug delivery and regulation of elevated intraocular pressure (IOP) in glaucoma patients.

[2006-0126]
\end{abstract}

Index Terms-Microflow control, microfluidics, microvalve, parylene.

\section{INTRODUCTION}

M ICROELECTROMECHANICAL systems (MEMS) is an enabling technology to build various microfluidic devices (e.g., channels, valves, pumps, mixers, etc.) for

Manuscript received June 28, 2006; revised September 1, 2006. This work was supported in part by the Engineering Research Centers Program of the National Science Foundation by NSF Award EEC-0310723. Subject Editor Y. Zobar.

P.-J. Chen and Y.-C. Tai are with the Department of Electrical Engineering, and the Division of Engineering and Applied Science, California Institute of Technology, Pasadena, CA 91125 USA (e-mail: pjchen@mems.caltech.edu).

D. C. Rodger is with the Department of Bioengineering, Division of Engineering and Applied Science, California Institute of Technology, Pasadena, CA 91125 USA. He is also with the Keck School of Medicine, University of Southern California, Los Angeles, CA 90033 USA

E. M. Meng is with the Department of Biomedical Engineering, Viterbi School of Engineering, University of Southern California, Los Angeles, CA 90089 USA.

M. S. Humayun are with the Keck School of Medicine, University of Southern California, Los Angeles, CA 90033 USA.

Y.-C. Tai is with the Department of Bioengineering, Division of Engineering and Applied Science, California Institute of Technology, Pasadena, CA 91125 USA.

Color versions of one or more of the figures in this paper are available online at http://ieeexplore.ieee.org.

Digital Object Identifier 10.1109/JMEMS.2006.889534

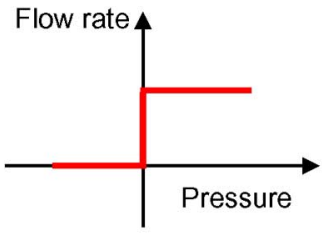

(a)

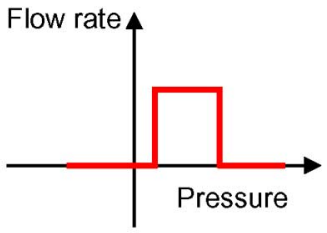

(b)
Fig. 1. Typical flow regulation profiles. (a) "Half-pass" regulation, in which constant flow rate is controlled with forward pressure loading. (b) "Bandpass" regulation, in which the flow is conducted only within a pressure loading range.

micro-total-analysis systems ( $\mu$ TAS) and lab-on-a-chip applications [1]-[3]. Microsystems incorporating such miniaturized devices can be utilized to do chemical/biochemical analysis, medical treatment, and drug delivery at the microscale. In order to fulfill systematic and reliable operation of these microfluidic systems, microflow regulation must be realized to control sample/buffer fluids. Traditionally off-chip flow regulators are used to maintain the consistency of the fluid source before introduction to these microsystems, but their size impedes miniaturization to a complete handheld system or bioimplant. Therefore, depending on the application, miniaturized flow regulators with various flow control profiles as shown in Fig. 1 have been developed and presented [4]-[7]. However, these paradigms are not fully compatible with conventional micromachining processes, resulting in challenges of integration with other components on a single chip. Accordingly, a novel microflow regulator consisting of surface-micromachined in-channel microvalves is proposed to achieve on-chip integration as well as effective flow regulation performance.

Microvalves are the key component to systematically control microflow for reliable operation [8]. They have the possibility of being integrated with other microfluidic components stemming from the fact that their mechanical structures are relatively simple. The valves are generally either active or passive, with passive valves (check valves) having the advantages of simple operation, zero power consumption, and ease of integration. Because check valves can shunt fluids with a single pressurized flow direction they are often recognized as "fluidic diodes." Fig. 2 shows the shunting behaviors of an electronic diode and a check valve [9]. Based on their actuation mechanism, micromachined check valves are categorized as normally open (Fig. 3(a), fluidic channel is always open unless there is sufficient backward pressure) [10] or normally closed (Fig. 3(b), fluidic channel is blocked until pressure is above cracking pressure) [11], [12] valves. However, according to Fig. 2, both types of check valves are not ideal. For normally open valves the 


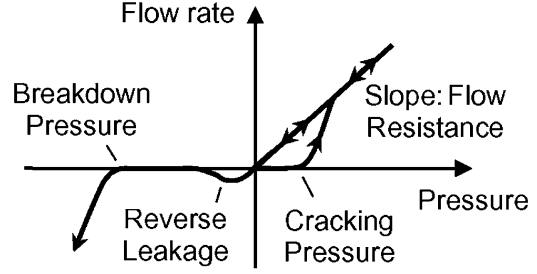

(a)

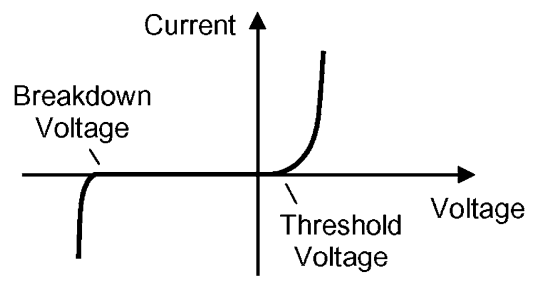

(b)

Fig. 2. Fluidic and electronic diodes. (a) Pressure/flow rate curve of a mechanical check valve. (b) $I-V$ curve of an electronic diode.

cracking pressure to conduct forward flow is minimal, while there is always a leakage problem when backflow is applied. For normally closed valves, the backflow leakage can be reduced, but the cracking pressure turns out to be so large that a reasonable flow rate is not achievable with low pressure loading. These problems are inevitable using current MEMS fabrication technologies.

Even though micromachined check valves are not ideal, different design schemes can be implemented to realize different fluidic behaviors from those in a single valve. In this paper, a novel dual-valve configuration has been developed to achieve passive microflow regulation as shown in Fig. 1(b) by incorporating a normally closed check valve and a normally open check valve together inside a microchannel. This configuration inherently regulates pressure and flow rate of fluids without any power consumption and greatly reduces the complexity of device fabrication and integration compared with other existing micro flow regulators. In addition to implementing similar design concepts from previous work [10], [11], both the design and fabrication of normally closed and normally open check valves have been modified and improved so that the in-channel dual-valved system is capable of controlling flows in a miniature regulation range [13]. This specific regulation range in a "bandpass" profile further favors biological conditioning in human health care. For example, a dual-valved device can be implanted in a glaucoma patient's eye to facilitate physical regulation of his or her elevated IOP. By shunting eye fluid in a specified pressure band, the implant protects the eye from hypotony by stopping drainage when the IOP is lower than the predetermined threshold point. It also prevents excessive eye fluid drainage during acute IOP spikes not due to glaucoma, such as when the eye is rubbed, pressed, hit. As used in drug delivery a dualvalved device can more accurately deliver a dose and prevent overdelivery of medication when the fluidic pressure is higher than the regular range [4]. Such a configuration can also be fully integrated in lab-on-a-chip systems for refined flow regulation in life science analysis and research.

\section{DESIGN}

In order to accomplish the target flow regulation profile as shown in Fig. 1(b), the back-to-back dual-valved configuration illustrated in Fig. 3(c) has been developed. This configuration requires only two in-channel check valves. By placing a normally closed valve in forward flow operation at the inlet and placing a normally open valve in backward flow operation at the outlet of a microchannel, the overall fluidic behavior of the dual-valved system combines the dashed regions of Fig. 3(a) and Fig. 3(b), resulting in the pressure/flow rate curve shown in Fig. 3(c). In this configuration, the normally closed check valve dominates the fluidic control in terms of conducting flows through the channel at pressures higher than the threshold point. The normally open check valve works in reverse leakage mode hence serving as a fluid stopper, in which flow is conducted at low pressures but blocked with applied pressures higher than the cut-off point. If the individual check valves are well-tuned, this passive in-channel dual-valved microsystem is able to spontaneously regulate pressure/flow rate in the designated range of forward flows in a "bandpass" profile without active actuation or power consumption. Additionally, this configuration is leak-proof to backward flows due to the characteristics of the normally closed valve at channel inlet. Because the dual valves must be integrated in-channel for this back-to-back operation, they were designed to be surface-micromachined for fabrication convenience.

Fig. 4 shows the designs of these microcheck valves. They are essentially thin-film structures microfabricated on a substrate. A free-standing membrane suspended by tethers acts as the normally open check valve, in which fluids can easily flow through in the forward direction while reserve leakage occurs in backflow [10]. This is because the membrane is suspended on top of the port orifice so that the flow is not blocked until backward pressure loading is applied to snap the membrane to the valve seat. The normally closed valve, on the other hand, needs a prestressing force to block the port orifice so forward flow can not pass through without sufficient pressure loading. Therefore, a vacuum-collapsed sealing method [11] was implemented to realize this valve behavior. Here, a valve membrane is connected to a surrounding ring chamber. When there is a sufficient pressure difference applied between the inside and outside of the chamber, the chamber top wall can be collapsed, resulting in the connected membrane being dragged downward and further pressed against the valve seat as long as the space between the valve and the valve seat is smaller than the chamber height. At this time flows can not be conducted unless the valve membrane is popped up with forward pressure loading, which realizes the dynamic behavior of a normally closed check valve. With sophisticated mechanical design, the check valves can be operated in harmony to achieve the desired microflow regulation capability. Because the crucial part of dual-valve operation is to ensure that the cracking pressure of the normally closed valve is well controlled, its mechanical design was verified by iterative FEMLAB (now called COMSOL Multiphysics) simulations to estimate the valve performance based on finite element method (FEM) [14]. As shown in Fig. 5, the ring chamber was first simulated to find the collapse point of valves of different dimensions 


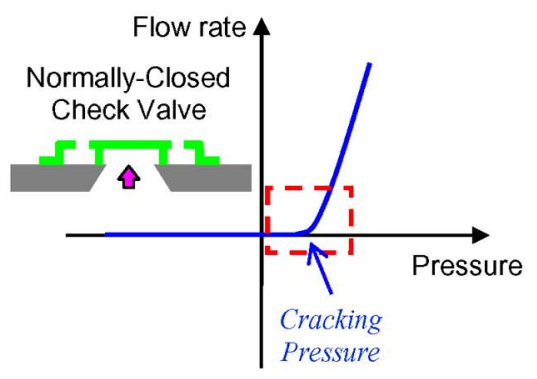

(a)

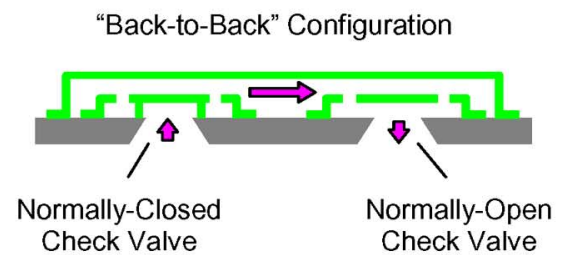

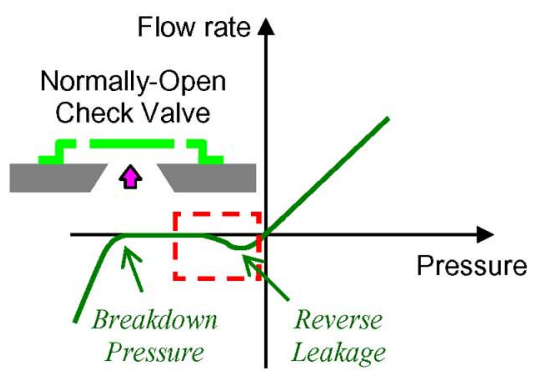

(b)

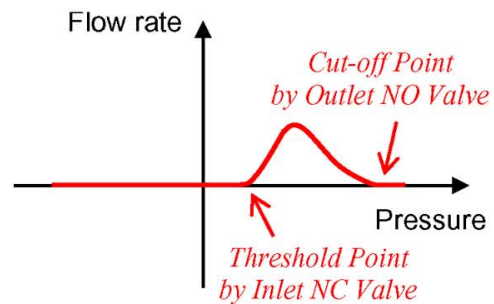

(c)

Fig. 3. Concept of dual-valved microflow regulation. Combining the single valve behavior shown in the dashed regions of (a) and (b) in a "back-to-back" configuration results in the passive "bandpass" pressure/flow rate regulation shown in (c). Block arrows in valve schematics denote the forward flow direction.

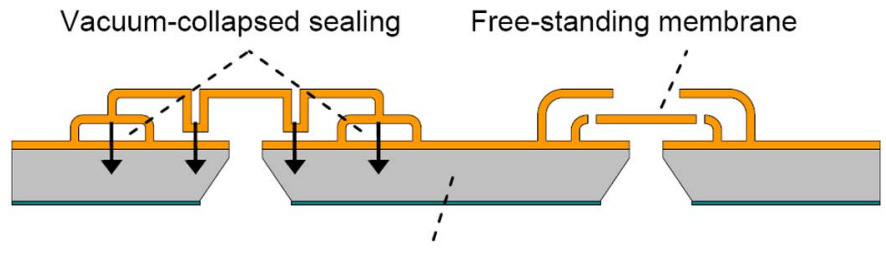

Valve seat with port orifices

Fig. 4. Cross section of normally closed (left) and normally open (right) microcheck valves. Arrows in normally closed valve denote the dragging direction when the chamber is collapsed by vacuum sealing, causing the connected valve membrane to be prestressed to the valve seat.

with a constant pressure difference (ambient pressure outside the chamber and vacuum inside the chamber). After the prestressing force with respect to height difference was obtained due to chamber collapse, it was used as a condition to simulate the required pressure loading to pop up the connected membrane. If the desired result was not attained then the dimensions of the valve structure (the chamber and the membrane) were changed and the simulation repeated. By such iterative simulation the behavior of the normally closed valve could be well estimated. Depending on dimensions and the use of straight-armed or twisted-armed tethers to suspend the membrane, single check valves can have different structural rigidities and their fluidic behaviors (flow resistance, cracking pressure, etc.) are correspondingly altered. As a result, microflow regulation with varying pressure/flow rate ranges can be virtually determined. Here the cracking pressure of the normally closed check valve was designed to be $10 \mathrm{mmHg}$ to realize the threshold point in the bandpass flow regulation profile.

Because the dual-valved system is proposed to achieve miniature pressure/flow rate regulation in life science applications, parylene $\mathrm{C}$ (poly-para-xylylene $\mathrm{C}$ ) was selected as the structural material due to its mechanical flexibility (Young's

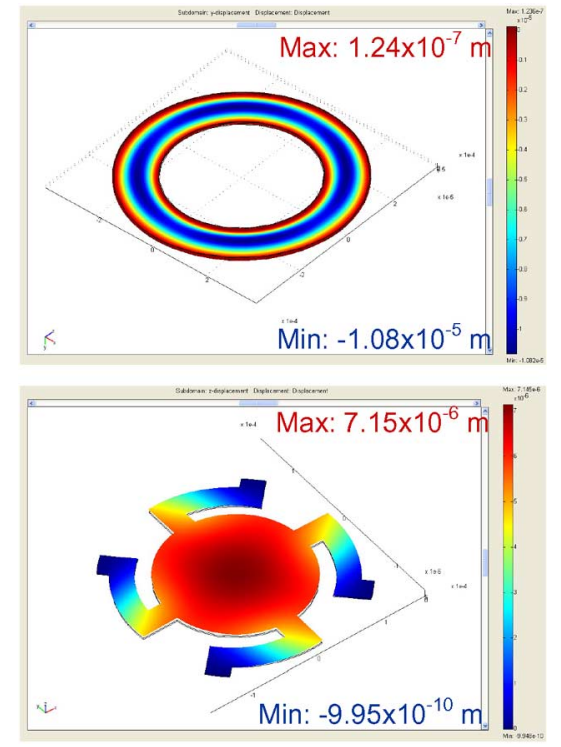

Fig. 5. FEMLAB simulations to estimate prestressing force of the vacuum-collapsed chamber (top) and resultant threshold pressure applied to valve membrane (bottom) to shunt fluids. By coupling these simulations the dynamic behavior of a complete normally closed check valve was determined. The resultant out-of-plane device deflections in the figures were obtained by using the dimensions shown in Table I under $10 \mathrm{mmHg}$ popping-up pressure loading.

modulus $\sim 4 \mathrm{GPa}$ ), chemical inertness, biocompatibility (USP Class VI grade), and CMOS/MEMS process compatibility [15], [16]. Furthermore, conformal parylene deposition takes place at room temperature in a vacuum ( $\sim 22$ mtorr $)$, which facilitates the vacuum-collapsed sealing of the normally closed check valve. Because parylene is much more flexible than poly-silicon and other conventional materials, the parylene-based microcheck valves ideally have higher compliance and better fluidic shunting performance compared to other thin-film 

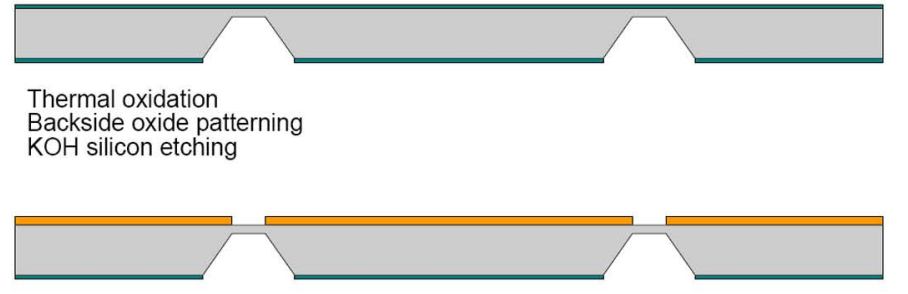

Frontside oxide removal

$1^{\text {st }}$ parylene deposition and patterning

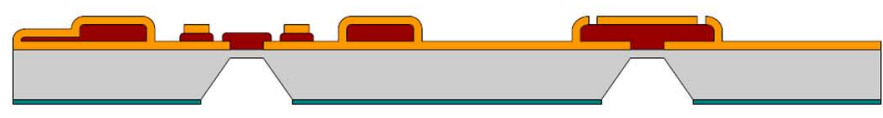

$1^{\text {st }}$ sacrificial photoresist coating and patterning

$2^{\text {nd }}$ parylene deposition and patterning
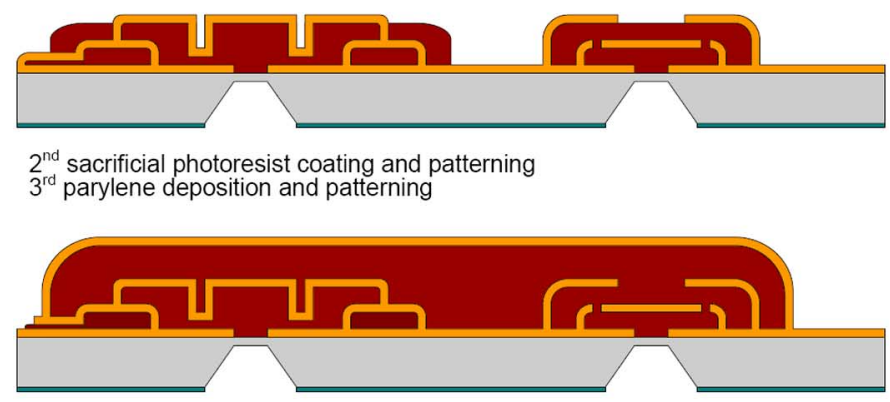

$3^{\text {rd }}$ sacrificial photoresist coating and patterning

$4^{\text {th }}$ parylene deposition and patterning

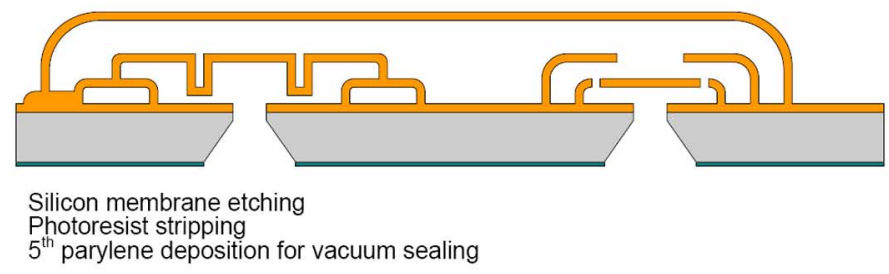

\section{$\square$ Silicon $\square$ SiO2 $\square$ Parylene $\square$ Photoresist}

Fig. 6. Fabrication process flow.

TABLE I

Design Metrics of the IN-ChanNel Dual VALVES IN THIS PAPER

\begin{tabular}{|c|c|}
\hline \multicolumn{2}{|c|}{ NORMALLY-CLOSED VALVE } \\
\hline Total width & $460 \mu \mathrm{m}$ \\
\hline Membrane diameter & $200 \mu \mathrm{m}$ \\
\hline Membrane thickness & $1.5 \mu \mathrm{m}$ \\
\hline Chamber width & $110 \mu \mathrm{m}$ \\
\hline Chamber height* & $7 \mu \mathrm{m}$ \\
\hline Chamber thickness* & $2.5 \mu \mathrm{m}$ \\
\hline $\begin{array}{c}\text { Critical space between } \\
\text { valve and valve seat }\end{array}$ & $5 \mu \mathrm{m}$ \\
\hline $\begin{array}{c}\text { Chamber etching/sealing } \\
\text { channel }\end{array}$ & $2 \mu \mathrm{m}$ \\
\hline Port orifice diameter & $100 \mu \mathrm{m}$ \\
\hline & *After final parylene vacuum sealing \\
\hline
\end{tabular}

valves [17], thus, accomplishing microflow regulations with high resolution. In addition, the devices comprise completely biocompatible/implantable materials so it is feasible to use them in biochemical/chemical analysis and human bioimplants. To enable more accurate design and estimation of the dual valves, the thin-film residual stress of parylene $\mathrm{C}[18]$ was also

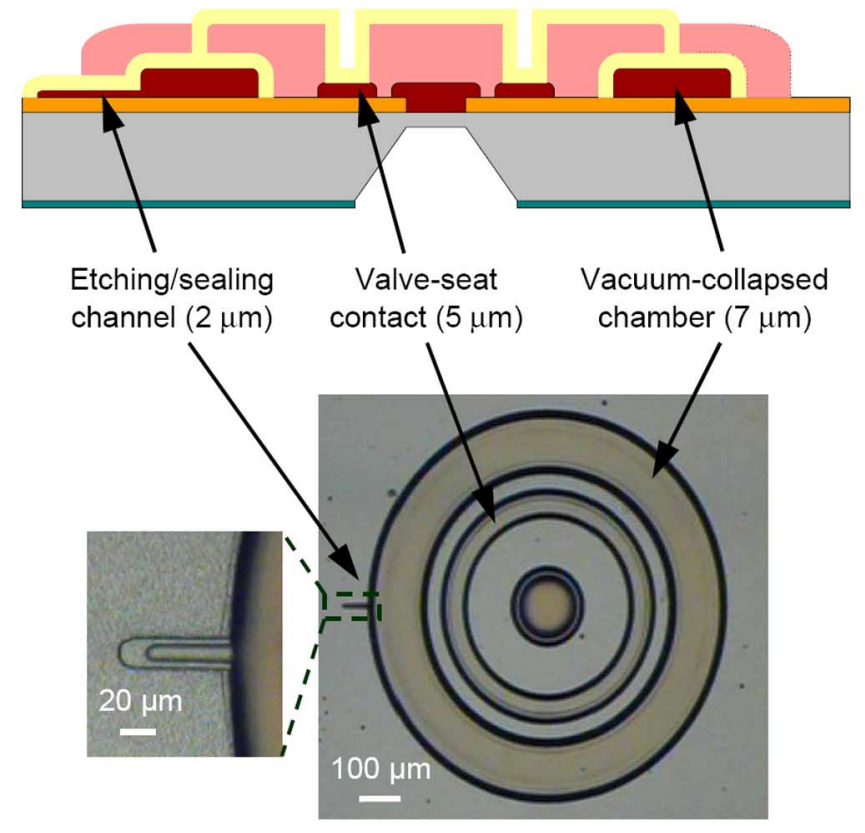

(a)

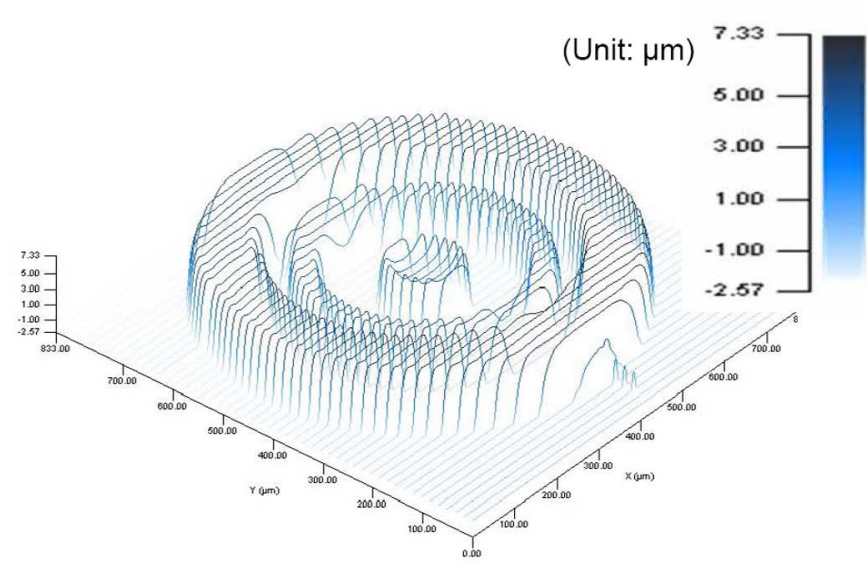

(b)

Fig. 7. Three-level sacrificial photoresist layer coating using three-step partial exposure lithography. (a) Cross-section schematic and top-view micrographs of the fabricated structures. (b) 3-D profile scan of (a).

considered in these simulations. Final design metrics of the in-channel dual valves are summarized in Table I.

\section{FABRICATION}

The fabrication process illustrated in Fig. 6 started with growing a $2-\mu \mathrm{m}$ wet oxide on a standard 4 " silicon wafer. The backside oxide was then patterned as a mask with buffered hydrofluoric acid (BHF) and the backside exposed silicon was bulk-etched with potassium hydroxide $(\mathrm{KOH})$ solution, leaving approximately $25-\mu \mathrm{m}$ membranes to define fluidic coupling ports. Ultimately these ports were used to provide fluidic access at the inlet and outlet of the microchannel. After removing the frontside oxide, the wafer was placed in air for some time to grow native oxide so that $0.5 \%$ saline A-174 (Specialty Coating System Inc., Indianapolis, IN) solution (DI 


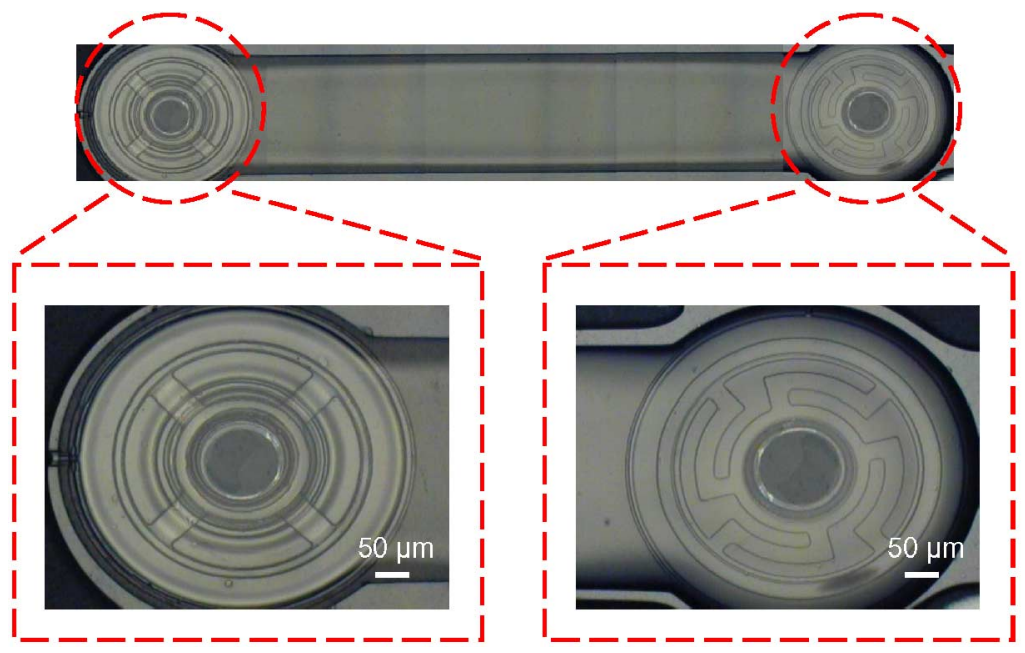

Fig. 8. Micrographs of the fabricated dual-valved channel ( $2 \mathrm{~mm}$ long in port-to-port distance) and cut-in views of the normally closed (left) and normally open (right) parylene check valves (top view). The diaphragm tethers can be designed as straight arms or twisted arms depending on performance requirements.

water:IPA:A-174 = 100:100:1 in volume) could be properly applied to promote adhesion between parylene $\mathrm{C}$ layer and the substrate. A multilayer surface-micromachining technology was subsequently utilized to build in-channel valve structures consisting of photoresist as sacrificial layers and parylene $\mathrm{C}$ as structural layers. AZ4620 (Clariant Corp., Charlotte, NC) was selected as the spin-coated photoresist material and parylene $\mathrm{C}$ deposition was conducted in a Cookson Electronics PDS 2010 system (Specialty Coating Systems Inc.). Details regarding dimensions of device features are noted in Table I. The sacrificial photoresist layers were hard-baked at $120{ }^{\circ} \mathrm{C}$ after lithography if applicable for edge-smoothening and degassing purposes. All parylene $\mathrm{C}$ layers were slightly roughened by a short period of oxygen plasma treatment in order to both reduce stiction [11] and promote adhesion in between each structural layer [19] in the fabricated devices. Cleaning procedures were also carefully taken on wafers before every parylene coating for stronger interlayer adhesion. Parylene layers were patterned by using an oxygen plasma etch with AZ4620 as the mask material to define device features. With these parylene processes, fabrication of the valve seats, check valves, and integrated microchannels was performed. After etching away the backside silicon membrane by either deep-reactive-ion etching (DRIE) or gas-phase $\mathrm{XeF}_{2}$ etching to open through holes and stripping photoresist with acetone, an additional frontside parylene $\mathrm{C}$ coating was finally conducted to accomplish the vacuum-collapsed sealing for the normally closed valve. Overall, the developed micromachining process includes five layers (four structural layers and one vacuum sealing layer) of parylene to completely fabricate the polymer-based dual-valved microfluidic system.

During processing the normally closed valve was the most complicated component because the height of the ring chamber and the spacing between valve membrane and valve seat have to be appropriately defined to realize vacuum-collapsed sealing. Consequently, as shown in Fig. 7, a three-step partial-exposure lithography was applied to a single sacrificial photoresist layer in order to construct a three-level base featuring a vacuum-collapsed ring chamber, a valve-seat contact, and a etching/sealing channel of a normally closed valve. In these structures, a $7-\mu \mathrm{m}$-high chamber was connected to a $2-\mu \mathrm{m}$-high channel for both final photoresist stripping and $1.5-\mu \mathrm{m}$-thick parylene vacuum sealing through that channel. A 5- $\mu \mathrm{m}$-thick photoresist spacer between the valve and the valve seat was also defined at the same time to control the prestressing force and threshold pressure loading of the fabricated valve by its height difference to the ring chamber. The 3-D profile scan taken with a P-15 surface profilometer (KLA Tencor Corp., San Jose, CA), as shown in Fig. 7(b), confirms that the three-level structures were fabricated as expected. Good planarization by spin-coating thick photoresist over the three-level structures was then required to create a flat valve membrane corresponding to uniform device performance. As for the normally open check valve, because it is designed to operate under reverse flow, a cover with a central orifice was placed on top of the valve to ensure the valve membrane encounters flows from above. Thus pressure loading can be properly applied as expected, which enhances the predictability of the reverse leakage behavior of the normally open valve. Fig. 8 shows the fabricated in-channel parylene dual valves. Channels with both single valves and dual valves were fabricated on-chip to experimentally characterize their behavior.

\section{EXPERIMENTAL SETUP}

Microfabricated in-channel parylene single check valves and dual valves were tested using the fluidic setup illustrated in Fig. 9. A customized testing jig featuring polyetheretherketones (PEEK) material was used to provide a macroscale/microscale fluidic interface with diced chips so that microflows could be introduced with regular tubing and fittings to the devices through their backside fluidic access ports [20], [21]. Both compressed air and water served as the fluid source for device characterization. On the front end of the jig, an off-chip pressure regulator and a pressure gauge were connected to control the injection pressure of the fluids. Although hydrostatic pressure generated by the gravitational effect of liquid column was not employed, the tuning resolution of the applied gauge pressure 


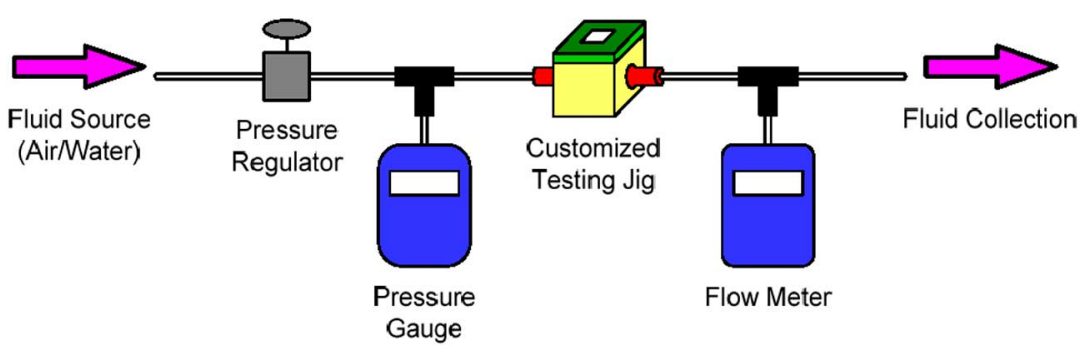

Fig. 9. Schematic of fluidic connection setup for device testing.

in this setup was up to $0.01 \mathrm{psi}(\sim 0.5 \mathrm{mmHg})$, which is sufficient for the desired regulation regime. Flow rate measurement and fluid collection was performed on the back end of the jig. Based on different viscosities of sample fluids, flow rate measurement was accomplished via different methods. Air flow rate was measured with an off-chip flow meter due to its large value $(\mathrm{mL} / \mathrm{min}$ range), while water flow rate was measured by recording the marching velocity of the water column front in the capillary due to its relatively small flow rates $(\mu \mathrm{L} / \mathrm{min}$ range). In marching velocity measurements, given the fact that the recording resolution was limited to $0.5 \mathrm{~mm}$ in marching distance and $1 \mathrm{~s}$ in marching time in this setup, the overall flow rate measurement error was approximately $5 \%-15 \%$ depending on the flow rate. Using these measurement techniques with the above considerations, the pressure/flow rate behavior of the devices for different fluids could be correctly characterized.

\section{RESULTS AND DISCUSSION}

Pressure/flow rate testing results of the micromachined in-channel valves are shown in Figs. 10 and 11 using air and water as the working fluids. Pressure readouts are presented in gauge pressure format to indicate the additional pressure loading from the injected fluids relative to atmospheric pressure. Incompressible flow was assumed for air flow measurements. These data show that air flow rate was much higher than water flow rate as expected due to their viscosity difference. The tested valves were all of the twisted-arm tether type in order to obtain more compliant deflection (mechanical response) with pressure loading compared to the straight-arm tether type [10]. In the following two sections, characterization results of the in-channel single valve and dual valve operation are described.

\section{A. Single Valve Testing}

Under single-valve operation, the fabricated normally closed check valve demonstrated a remarkably low cracking pressure of approximately $0.2 \mathrm{psi}(\sim 10 \mathrm{mmHg})$ for both air and water flow as shown in Figs. 10(a) and 11(a). This is a result of the fluidic pressure being applied on the central area of the flexible membrane, thus, effectively overcoming the prestressing force of the vacuum-collapsed chamber and shunting the fluids to the microchannel. Lower cracking pressure was obtained with air than with water as the fluid, implying that air is generally easier to shunt than water because a higher interfacial disturbance (e.g., capillary effect) appeared at the hydrophobic parylene $\mathrm{C}$ surface. As a result, additional pressure loading is required for water to flow in parylene channels. In backward operation, there was no reverse leakage occurring in the normally

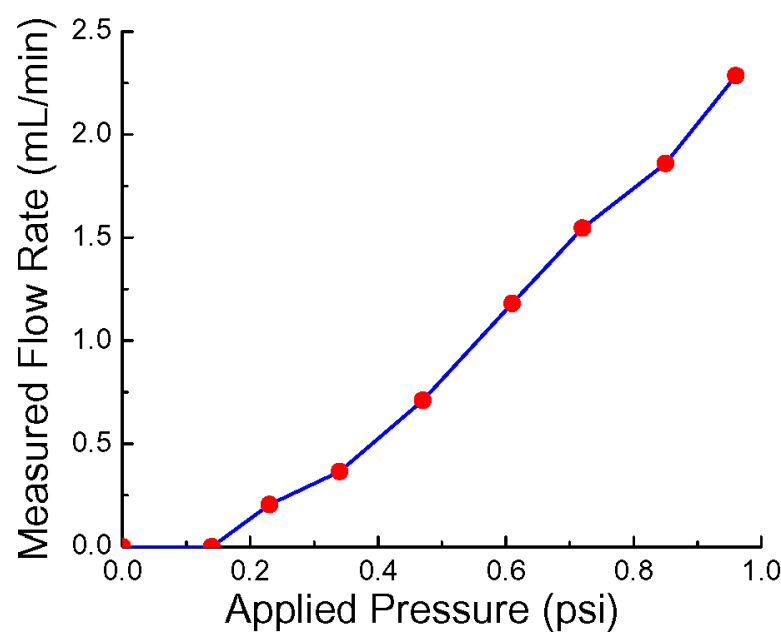

(a)

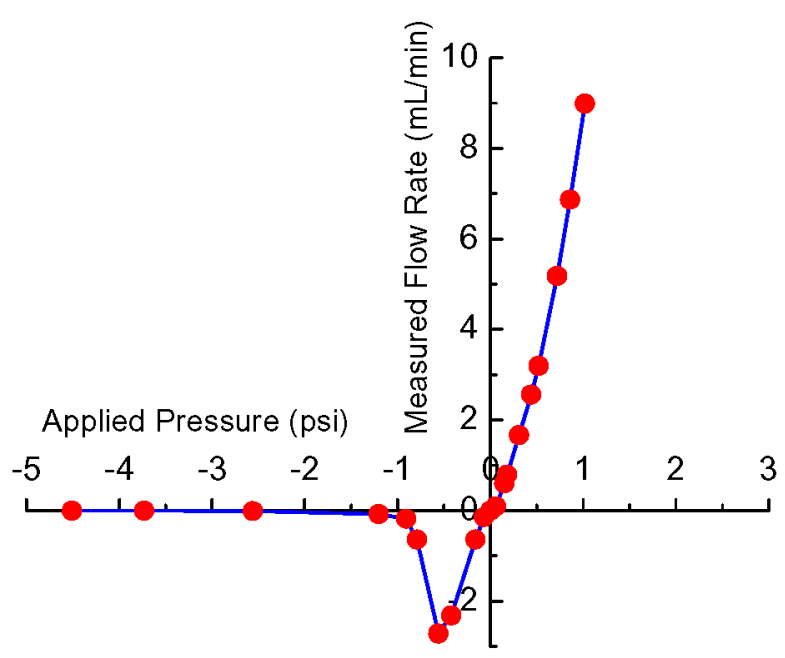

(b)

Fig. 10. Single valve operation with air as the working fluid. (a) Pressure/flow rate curve of the normally closed check valve. (b) Pressure/flow rate curve of the normally open check valve.

closed valve with pressure loading greater than $25 \mathrm{psi}$, close to the maximum limit of the off-chip regulator and pressure gauge in the testing setup. For the normally open check valve, as shown in Figs. 10(b) and 11(b), no obvious cracking pressure effect was observed and a higher flow rate was obtained with the same degree of pressure loading. Given the fact that the valve membrane designs in the normally open valve and the normally closed valve were different, the forward flow resistance of the normally 
TABLE II

Comparison of Reported Parylene Microcheck Valves (With Water as the Working Fluid)

\begin{tabular}{|c|c|c|c|c|c|}
\hline & Ref. [10] & Ref. [11] & Ref. [12] & This work & This work \\
\hline Valve type* $^{*}$ & NO & NC & NC & NO & NC \\
\hline On-chip placement & No channel & In-channel & No channel & In-channel & In-channel \\
\hline Tether type & Twisted-arm & Center anchor & Straight-arm & Twisted-arm & Twisted-arm \\
\hline Occupied area $^{\dagger}$ & $10^{-7} \mathrm{~m}^{2}$ & $10^{-8} \mathrm{~m}^{2}$ & $10^{-8} \mathrm{~m}^{2}$ & $10^{-7} \mathrm{~m}^{2}$ & $10^{-7} \mathrm{~m}^{2}$ \\
\hline Membrane thickness & $2 \mu \mathrm{m}$ & $0.7 \mu \mathrm{m}$ & $0.5 \mu \mathrm{m}$ & $1.5 \mu \mathrm{m}$ & $1.5 \mu \mathrm{m}$ \\
\hline Cracking pressure & $<0.1 \mathrm{psi}$ & $\sim 2.9 \mathrm{psi}$ & $\sim 1 \mathrm{psi}$ & $<0.1 \mathrm{psi}$ & $\sim 0.2 \mathrm{psi}$ \\
\hline $\begin{array}{c}\text { Pressure to stop } \\
\text { reverse leakage }\end{array}$ & Not reported & 0 & 0 & $\sim 1.5 \mathrm{psi}$ & 0 \\
\hline Breakdown pressure & $>87 \mathrm{psi}$ & $\sim 39 \mathrm{psi}$ & $>30 \mathrm{psi}$ & $>25 \mathrm{psi}$ & $>25 \mathrm{psi}$ \\
\hline
\end{tabular}

$* \mathrm{NO}=$ normally-open $\mathrm{NC}=$ normally-closed

${ }^{\dagger}$ Calculated order of scale

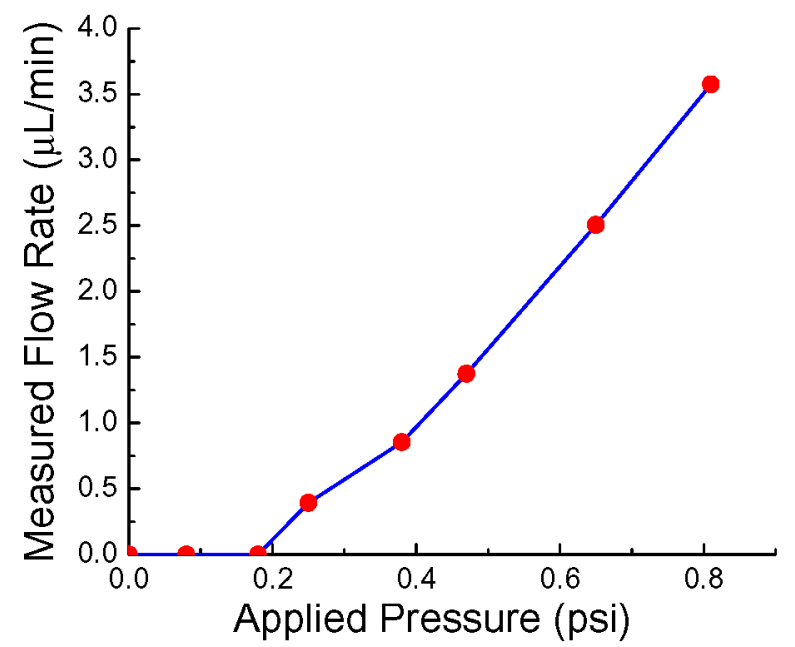

(a)

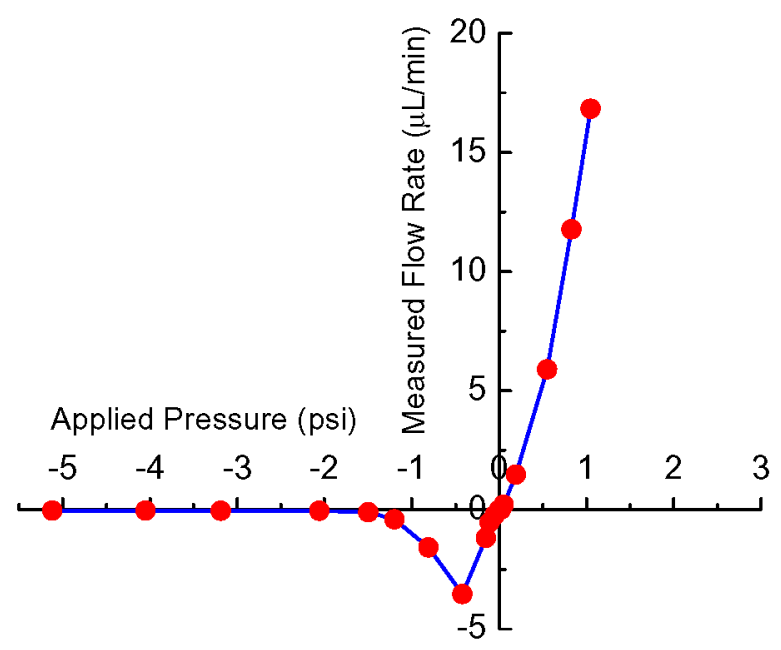

(b)

Fig. 11. Single valve operation with water as the working fluid. (a) Pressure/flow rate curve of the normally closed check valve. (b) Pressure/flow rate curve of the normally open check valve.

open valve was significantly lower compared to that of the normally closed valve. In terms of reverse leakage, the backward flow rate was obtained within $1.5 \mathrm{psi}(\sim 80 \mathrm{mmHg})$ with a peak at more than $2 \mathrm{~mL} / \mathrm{min}$ for air flow and $2 \mu \mathrm{L} / \mathrm{min}$ for water flow.
This leak rate started to decrease when the pressure loading was higher than $0.6 \mathrm{psi}(\sim 30 \mathrm{mmHg})$ and was greatly suppressed with pressure loading higher than 1 psi ( $~ 50 \mathrm{mmHg})$, which implies that the valve membrane was in firm contact with valve seat. The breakdown pressure was confirmed at more than $25 \mathrm{psi}$ with no obvious leak rate within this range of pressure operation. Even though a wide variety of designs of different dimensions were implemented, some figures of merit of the single in-channel parylene valves were compared with reported parylene microcheck valves [10]-[12] in Table II to briefly describe the improvement of the valves in this paper.

\section{B. Dual Valve Testing}

Miniature pressure/flow rate regulation was successfully performed in dual valve operation. Fig. 12 shows that, by combining the behaviors of the single check valves, the dual-valved microsystem was able to regulate pressure in the range of $0-1 \mathrm{psi}$ ( 0-50 $\mathrm{mmHg}$ ) with measured flow rates of up to $0.7 \mathrm{~mL} / \mathrm{min}$ for air flow and $1.3 \mu \mathrm{L} / \mathrm{min}$ for water flow. Because more microfluidic elements were placed in dual valve configuration than in single valve configuration, a large fluidic resistance obtained by the dual check valves with a microchannel results in relatively small flow rates. As expected, no reverse leakage was observed with pressure loading greater than 25 psi.

The micromachined in-channel parylene valves were successfully characterized with reliable and repeatable behaviors from the same device in cyclic operation regardless of errors in gauge pressure and marching velocity/flow rate measurements. However, their performance unavoidably had some extent of variation. For example, the measured cracking pressure of the normally closed check valves from the same batch of fabrication varied from 8 to $13 \mathrm{mmHg}$ based on the tested devices in this paper, while still being in good agreement with the designed value of the popping-up pressure loading from the FEMLAB simulations. The following three factors related to the fabrication process contributed to the resultant variation: 1) thickness control of photoresist coating; 2) thickness control of parylene $\mathrm{C}$ deposition; and 3) material property control of thin-film parylene $\mathrm{C}$. Consequently, more process calibration and characterization should be conducted in order to enhance uniformity of the device performance so that the bandpass pressure regulation range can be precisely controlled depending on specific application. 


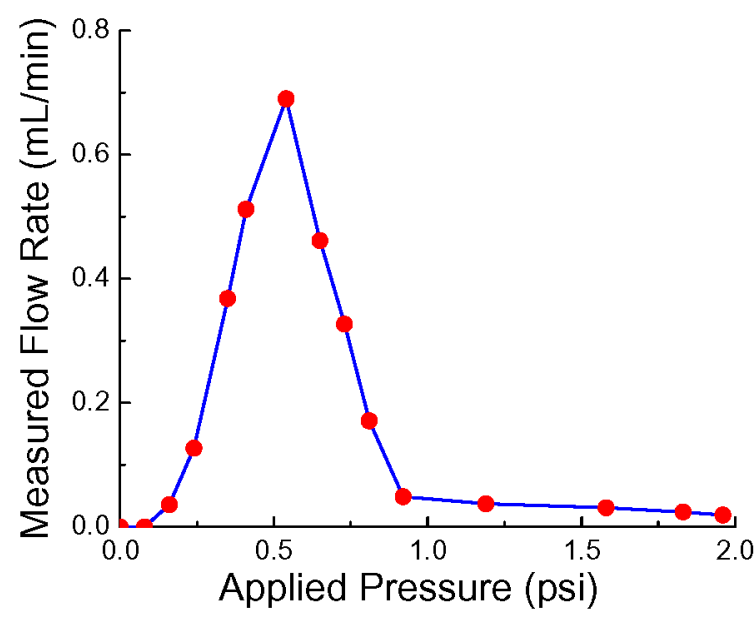

(a)

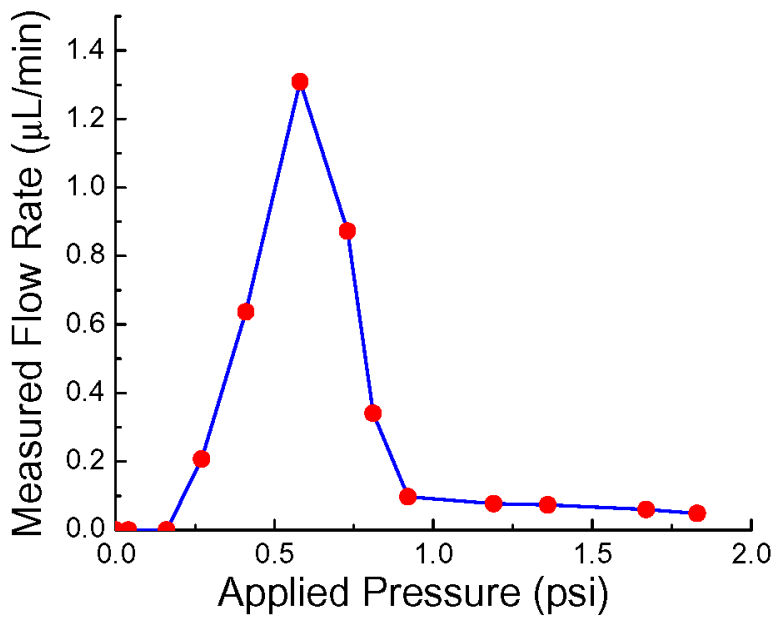

(b)

Fig. 12. Dual valve operation for miniature microflow regulation. (a) Air flow regulation. (b) Water flow regulation.

\section{CONCLUSION}

A parylene-based unpowered dual-valved microflow regulation system has been successfully developed. By incorporating a normally closed and a normally open check valve in back-toback configuration inside a microchannel, fluid pressure/flow rate can be systematically controlled. Because of the passive operation of the check valves, only mechanical elements were involved and no power consumption/transduction was required. A multilayer surface-micromachining technology was applied to construct the integrated dual-valved devices. Fabrication and characterization of single check valves and dual valves have been demonstrated. In single valve testing, both normally closed and normally open check valves achieved fluidic behaviors in good agreement with design and simulation. Excellent miniature pressure/flow rate regulation was obtained in dual valve testing. Flow rates that peaked at $0.7 \mathrm{~mL} / \mathrm{min}$ for air flow and $1.3 \mu \mathrm{L} / \mathrm{min}$ for water flow were obtained within a $0-50 \mathrm{mmHg}$ pressure range. With this unique microflow regulation performance, our biocompatible and implantable dual-valved system can be utilized for many applications in lab-on-a-chip systems for life science research, such as drug delivery and regulation of the elevated IOP of glaucoma patients.

\section{ACKNOWLEDGMENT}

The authors especially thank T. Roper for his fabrication assistance.

\section{REFERENCES}

[1] A. V. d. Berg and T. S. J. Lammerink, "Micro total analysis systems: Microfluidic aspects, integration concept and applications," Microsyst. Technol. Chem. Life Sci. Topics Curr. Chem., vol. 194, pp. 21-49, 1998.

[2] S. Shoji, "Fluids for sensor systems," Topics Curr. Chem., vol. 194, pp. 163-188, 1998.

[3] T. Vilkner, D. Janasek, and A. Manz, "Micro total analysis systems. recent developments," Analyt. Chem., vol. 76, pp. 3373-3385, 2004.

[4] P. Cousseau, R. Hirschi, B. Frehner, S. Gamper, and D. Maillefer, "Improved micro-flow regulator for drug delivery systems," in Proc. 14th IEEE Int. Conf. MEMS 2001, Interlaken, Switzerland, Jan. 21-25, 2001, pp. 527-530.

[5] B. Yang, J. W. Levis, and Q. Lin, "A PDMS-based constant-flowrate microfluidic control device," in Proc. 17th IEEE Int. Conf. MEMS 2004, Maastricht, The Netherlands, Jan. 25-29, 2004, pp. 379-382.

[6] J. Collier, D. Wroblewski, and T. Bifano, "Development of a rapid-response flow-control system using MEMS microvalve arrays," J. Micoelectromech. Syst., vol. 13, pp. 912-922, 2004.

[7] A. Debray, T. Nakakubo, K. Ueda, S. Mogi, M. Shibata, and H. Fujita, "A passive micro gas regulator for hydrogen flow control," J. Micromech. Microeng., vol. 15, pp. 202-209, 2005.

[8] K. W. Oh and C. H. Ahn, "A review of microvalves," J. Micromech. Microeng., vol. 16, pp. 13-39, 2006.

[9] X. Q. Wang, "Integrated parylene micro electro mechanical systems (MEMS)," Ph.D. dissertation, Calif. Inst. Technol., Pasadena, 2000.

[10] X. Q. Wang, Q. Lin, and Y.-C. Tai, "A parylene micro check valve," in Proc. 12th IEEE Int. Conf. MEMS 1999, Orlando, FL, USA, Jan. 17-21, 1999, pp. 177-182.

[11] X. Q. Wang and Y.-C. Tai, "A normally-closed in-channel micro check valve," in Proc. 13th IEEE Int. Conf. MEMS 2000, Miyazakli, Japan, Jan. 23-27, 2000, pp. 68-71.

[12] J. Xie, X. Yang, X. Q. Wang, and Y.-C. Tai, "Surface micromachined leakage proof parylene check valve," in Proc. 14th IEEE Int. Conf. MEMS 2001, Interlaken, Switzerland, Jan. 21-25, 2001, pp. 539-542.

[13] P.-J. Chen, D. C. Rodger, E. Meng, M. S. Humayun, and Y.-C. Tai, "Surface-micromachined in-channel parylene dual valves for unpowered microflow regulation," in Proc. 12th Solid State Sens., Actuators, and Microsyst. Workshop, Hilton Head, SC, Jun. 4-8, 2006, pp. 205-208.

[14] A. Ilzhofer, B. Ritter, and C. Tsakmakis, "Development of passive microvalves by the finite element method," J. Micromech. Microeng., vol. 5, pp. 226-230, 1995.

[15] Specialty Coating Systems [Online]. Available: http://www.scscoatings.com/parylene_knowledge/specifications.cfm

[16] Certificate of Compliance, USP Biological Tests. Northwood, $\mathrm{OH}$ : North Amer. Sci. Assoc., Inc.

[17] D. C. S. Bien, S. J. N. Mitchell, and H. S. Gamble, "Fabrication and characterization of a micromachined passive valve," J. Micromech. Microeng., vol. 13, pp. 557-562, 2003.

[18] T. A. Harder, T.-J. Yao, Q. He, C.-Y. Shih, and Y.-C. Tai, "Residual stress in thin-film parylene C," in Proc. 15th IEEE Int. Conf. MEMS 2002 , Las Vegas, NV, Jan. 20-24, 2002, pp. 435-438.

[19] J. Xie, J. Shih, Q. Lin, B. Yang, and Y.-C. Tai, "Surface micromachined electrostatically actuated micro peristaltic pump," Lab on a Chip, vol. 4, pp. 405-501, 2004.

[20] Q. He, C. Pang, Y.-C. Tai, and T. D. Lee, "Ion liquid chromatography on-a-chip with beads-packed parylene column," in Proc. 17th IEEE Int. Conf. MEMS 2004, Maastricht, The Netherlands, Jan. 25-29, 2004, pp. 212-215.

[21] C.-Y. Shih, Y. Chen, J. Xie, Q. He, and Y.-C. Tai, "On-chip temperature gradient interaction chromatography," J. Chromatogr. A, vol. 1111, pp. 272-278, 2006. 


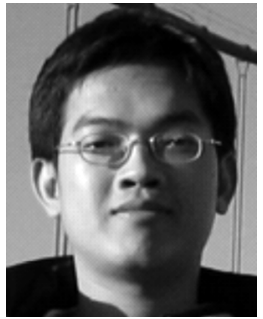

Po-Jui Chen (S'03) was born in Taipei, Taiwan, in 1980. He received the B.S. degree in mechanical engineering from the National Taiwan University, Taipei, Taiwan, in 2002, and the M.S. degree in electrical engineering from the California Institute of Technology (Caltech), Pasadena, in 2004, and currently, he is working towards the Ph.D. degree in electrical engineering in the area of MEMS at the Caltech Micromachining Laboratory.

He joined Dr. Y.-C. Tai's Caltech Micromachining Laboratory as a Graduate Researcher in 2003 . He has authorized more than ten peer-reviewed journal and conference papers. His research interests include implantable biomedical microdevices, integrated microfluidic systems, micro-total-analysis systems (TAS), MEMS sensors/actuators/structures, and micro/nanofabrications.

Mr. Chen received several scholarships, the Sorenson Fellowship, the outstanding college youth award at National Taiwan University in 2002, and was elected as an honorary member of the Phi Tau Phi scholastic honor society in Taiwan in 2002. He is the recipient of the Student Poster Award in the International TAS 2006 Conference in Tokyo, Japan. He is a student member of the American Society Of Mechanical Engineers (ASME) and the Association for Research in Vision and Ophthalmology (ARVO) and a full member of Sigma $\mathrm{Xi}-$ the scientific research society.

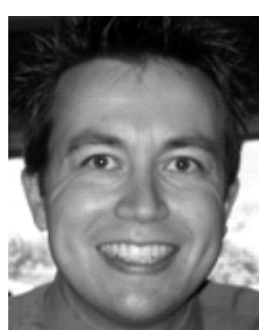

Damien C. Rodger (S'97) received the B.S. degree in electrical engineering (magna cum laude with honors) from Cornell University, Ithaca, NY, in 2000. Currently, he is working towards the M.D./Ph.D. degree at the Keck School of Medicine, the University of Southern California, Los Angeles, and the California Institute of Technology, Pasadena, conducting research in bioengineering under Dr. Y.-C. Tai and Dr. M. Humayun in the area of bioMEMS for ophthalmic use.

His MEMS research while at Cornell University concentrated on SCREAM-fabricated microwave transmission lines and continuous phase shifters, conducted under Dr. N. MacDonald, and on biomotordriven microdevices as a W.M. Keck Foundation Fellow in nanobiotechnology under Dr. C. Montemagno. He also worked in the MEMS group at the NASA Jet Propulsion Laboratory on the Coriolis force vibratory microgyroscope, under Dr. T. Tang and Dr. W. Tang.

Mr. Rodger held a Whitaker Foundation Graduate Fellowship in Biomedical Engineering from 2003 to 2006. He a student member of the Association for Research in Vision and Ophthalmology (ARVO).

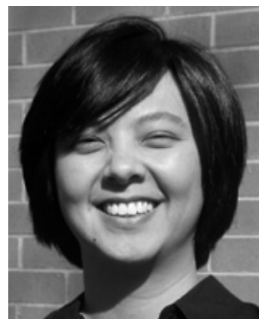

Ellis M. Meng (M'02) received the B.S. degree in engineering and applied science and the M.S. and Ph.D. degrees in electrical engineering from the California Institute of Technology (Caltech), Pasadena, in 1997, 1998, and 2003, respectively.

She joined the Biomedical Engineering Department at the University of Southern California, Los Angeles, in summer 2004. Her research interests include bioMEMS, implantable biomedical microdevices, microfluidics, multimodality integrated microsystems, and packaging. Currently, she is a
Researcher in the National Science Foundation Biomimetic MicroElectronic Systems Engineering Research Center (BMES ERC) in which she is a Thrust Leader for Interface Technology and the Associate Director of Education and Student Diversity.

Dr. Meng was a recipient of the Intel Women in Science and Engineering Scholarship, Caltech Alumni Association Donald S. Clark Award, and Caltech Special Institute Fellowship. She is a recipient of the NSF CAREER award and a member of Tau Beta Pi, the American Society Of Mechanical Engineers (ASME), the Society of Women Engineers (SWE), and the Association for Research in Vision and Ophthalmology (ARVO).

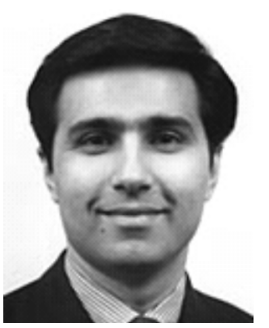

Mark S. Humayun (M'97) received the B.S. degree from Georgetown University, Washington, DC, in 1984, the M.D. degree from Duke University, Durham, NC, in 1989, and the Ph.D. degree from the University of North Carolina, Chapel Hill, in 1994.

$\mathrm{He}$ finished his training by completing an Ophthalmology Residency at Duke University and a Fellowship in Vitreoretinal diseases at The Johns Hopkins Hospital. He stayed on as faculty at The Johns Hopkins Hospital while he became the Associate Professor. Currently, he is a Professor of Ophthalmology, Biomedical Engineering, and Cell and Neurobiology at the University of Southern California, Los Angeles. He is the Director of the National Science Foundation Biomimetic MicroElectronic Systems Engineering Research Center (BMES ERC). He is the Co-Developer of a retinal implant that has received wide attention for its potential to restore sight. He is also the Director for the Department of Energy Artificial Retina Project that is a consortium of five Department of Energy labs and four universities, as well as industry. His research interests include retinal prostheses, implantable microelectronics, and ophthalmic surgical instrumentation.

Dr. Humayun is a member of the Biomedical Engineering Society, the Association for Research in Vision and Ophthalmology (ARVO), the Vitreous Society, and the Retina Society.

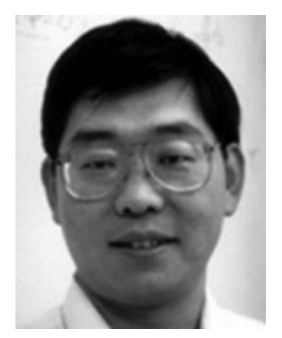

Yu-Chong Tai (M'97-SM'03-F'06) received the B.S. degree in electrical engineering from the National Taiwan University, Taipei, Taiwan, in 1981 and the M.S. and Ph.D. degrees in electrical engineering from the University of California at Berkeley in 1986 and 1989, respectively.

He joined the Faculty of Electrical Engineering at the California Institute of Technology (Caltech), Pasadena, where he built the Caltech Micromachining Laboratory and currently is a Professor of Electrical Engineering, Mechanical Engineering, and Bioengineering. Hi current research interests include flexible MEMS, bioMEMS, MEMS for retinal implants, parylene-based integrated microfluidics, neuroprobes/neurochips, and HPLC-based lab-on-a-chip systems.

Dr. Tai received several awards such as the IBM fellowship, the Best Thesis Award, the Presidential Young Investigator (PYI) Award and the David and Lucile Packard Fellowship. He Co-Chaired the IEEE International MEMS 2002 Conference, Las Vegas, NV. 